des Uterus eingesetzt hatte. Das Körnchen wuchs und verbreiterte sich. Nicht lange nachher war die ganze Schleimhaut der vorderen Lippe und der ganzen vorderen Wand des Halses mit körnig sich anfühlenden Massen besetzt und infiltrirt. Die hintere Lippe war frei. Am linken Lippenwinkel sass ein isolirter haselnussgrosser Knoten. Ein kleinerer Knoten fand sich im rechten Vaginalgewölbe, in inniger Berührung mit der vorderen Lippe. In der Epikrise über diesen Fall bemerkt Spiegelberg: ,Alle diese neuen Infiltrationen hatten denselbęn Bau, wie die erste Erkrankung, und es ist gewiss höchst wahrscheinlich, dass die, den lethalen Ausgang einleitenden Erkrankungen an der vorderen Muttermundlippe und am Vaginalgrunde durch directe Impfung des Gewebes mit Geschwulstpartikelchen mittels der bei der zweiten Operation am 20. Januar gesetzten Verletzungen an jenen Stellen bedingt war."

Wenn nun auch in unserem Falle die mikroskopischen Bilder nicht genau dem primären Sarcome ähnelten, sondern nur einige Stellen einen Bau zeigten, der mit der intrauterinen Geschwulst verglichen werden konnte, so glaube ich doch, aus den oben angeführten Grïnden annehmen zu müssen, dass wir es bier mit einer Einimpfung zu thun hatten.

\title{
Retrorectale Dermoidcyste.
}

Ein Beitrag zur Casuistik der Tumoren des Unterleibes.

$$
\text { Von }
$$

Dr. A. Deahna,

Assistenzarzt der ehirurg. Klinik in Freiburg.

Am 16. Angust 1873 wurde das 18 jährige Dienstmädchen S. H. ans $D$. in die hiesige Klinik aufgenommen. Seit ihrem 13. Jahre menstruirt, zum letzten Male vom 2.-6. August, war sie früher stets gesund gewesen, bis sie am Abend des 11. Aug. von heftigen, ziehenden und spannenden Schmerzen im Unterleibe befallen wurde und die ganze Nacht schlaflos zubrachte. Nichtsdestoweniger ging. sie am folgenden Morgen wieder an ihre Arbeit, wurde aber bald während derselben in der Küche am Herd von furchtbaren Schmer- 
zen im Leibe befallen, so dass sie ohnmächtig umfiel und an Bett geschafft werden musste. Sie erbrach dann mehrmals hintereinander, den Urin kounte sie nur nach heftigem Drängen entleeren, Stuhlgang exfolgte erst auf Anwendung abführender Mittel, der Appetit mangelte vollständig, der Leib war sehr empfindlich, stark aufgetrieben und hart anzufühlen. So blieben im Allgemeinen die Verhältnisse in den xächstfolgenden Tagen, bis sie am 16. Ang. im Spital Hülfe suchte.

Ihr Zustand bei der Aufuahme war folgender: Patientin von kleiner Statur, kräftigem Knochenbau, Schleimhäute anämisch, Wangen blass, Halz kurz, dick, Struma geringen Grades. Lungen und Herz zeigen normales Verhalten.

Das Abdomen ist im Nivean des Thorax, der untere Theil, vom Nabel abwärts, prominirt und ist leicht kugelig gewölbt. Aut dem ganzen Abdomen ist rechts und links lauter tympanitiseher PercussionsSchall, mit Ausnahme der mittleren Unterbauch - und Nabelgegend, wo leerer Sehall ist, der etwas weiter nach links, als nach rechts hinüber reicht. Im Bezirke des leeren Schalles ist bedeutende Vermehrung der Resistenz; Urin hat Patientin seit längerer Zeit nicht entleeren können. - Nach der Entleerung der Blase mit den Katheter verkleinert sich der Beairk des leeren Schalles und es ist jetzt, wegen geringer Spannung der Bauchdecken, möglich, den Unterleib zu palpiren. Man fühlt links an der hinterwand des grossen Beckens bis zur Mitte zwischen Nabel und Symphyse einen harten, derben Korper, der sich nach unten nicht abgrenzen lässt. Bei der Lnterguchung per vaginam gelangt man mit dem Finger bis wenig über die Symphyse, weiter nach aufwärts ist die hintere Vaginalwand nach rorn gedrängt, wodurch eine spaltförmige Verengerung der Scheide entsteht, welche dem weiteren Vordringeu des Fingers bis zum Erreichen des Uterus ein Ziel setzt. Weiteren Aufschluss verschafft die FingerUntersuchung durch das Rectum. Zwar kann man auch hier nicht viel weiter, als bis zur Höhe der Symphyse gelangen, allein man fiuhlt doch mit grösster Deutlichkeit eine Geschwulst, die hinter dem Rectum liegt, sich derb elastisch anfühlt und an dea oberen Partieen weniger resistent ist, als an den unteren; nach unten reicht sie bis nahe an den After, dem sie durch die Wirkung der Bauclipresse noch mehr genähert werden kann; das Rectum wird von der Geschwulst nach vorn und rechts verdrängt, so dass es zu einem halbkreisformigen Spalte zusammengedrückt ist; die obere Grenze lässt sieh mit dem f'inger nicht erreichen. Bei Druck auf die Bauchdecken wird die Geschwulst stärker angespannt und lâsst sich ein wenig nach unten verschieben, umgekehrt kann man sie bei Druck von unten nach oben schieben, die Beweglichkeit ist aber nur eine sehr'geringe. Ueber einen etwaigen Zusammenhang mit dem Uterus konnte man keinen Aufschlass gewinnen, da derselbe weder durch die Banchdecken, noch vou Vagina oder Rectum her erreiehbar wax; nur soviel liess sich mit Bestinntheit sagen, dass die Geschwulst nicht der retrovertirte schwangere Uterus selbst war, da Patientin exst 6 Tage 
vor ihrer Erkrankung zum letzten Male menstruirt war und auch sonst kein Zeichen der Gravididät vorlag, ausserdem es nicht leicht denkbar ist, dass der retrovertirte Uterus sich hinter das Rectum herabsenken sollte; gegen die Annahme einer Cyste des Ovariums mit serösem oder sonst wie beschaffenem Inhalt sprach gleichfalls die Lage hinter dem Rectum und die verhältnissmässig geringe Beweglichkeit, ohne dass peritonitische Erscheinungen vorausgegangen wären. Es konnte also kaum etwas Anderes vorliegen, als eine Cysten-Geschwulst, die sich selbstständig zwischen Rectum und Kreuzbein entwickelt hatte, eine sichere Diagnose konnte aber erst nach einer ProbePunction gestellt werden, welche sofort mit einem sehr feinen Troieart vom. Rectum ats vorgenommen wurde; es entleeren sich circa 30 Gramm einer dünnen, geruchlosen Flüssigkeit von gelblicher Farbe und amphigener Reaction, in der eine Menge gelber und braungrüner, krümeliger Massen enthalten sind, die sich unter dem Mikroskop als Epidermiszellen darstellen, durch welchen Befund der Tumor mit Sicherheit als Dermoid.Cyste diagnosticirt werden konnte. Die Reaction auf die Punction war eine sehr unbedeutende; die Beschwerden blieben mit geringen Remissionen, wie sie vor der Punction waren, weil wegen bald eingetretener Undurchgängigkeit der Canüle blos eine geringe Menge des Inhalts hatte ausfliessen können. Bald aber drängten die beinahe vollständige Urinretention und die Schmerzbaftigkeit der Katheterisation zu einer gründlichen Abhülfe. Es wurde deshalb am 30. August eine neue Punction vom Rectum aus mit einem starken Troicart vorgenommen und mit dem Aspirateur von Dieulafoy eine Ménge von $220 \mathrm{Ccm}$. Flüssigkeit herausgezogen, leider wurde die Canüle wieder frühzeitig durch Epidermispfröpfe verstopft. Während nun der durch die Probepunction herausbeförderte Cysteninhalt geruchlos war und amphigen reagirte, zeigte der jetzige sehr schlechten Geruch und intensiv saure Reaction. Die Flüssigkeit besteht makroskopisch aus einem hellbraunen, ins Graugrüne schillernden Menstruum, in welchem stecknadelkopf- bis linsengrosse, derbe, weisse Schollen, maulbeerartige, theils weisse, theils bräunliche kleine Kügelchen, und endlich verfilzte Haarpfröpfe herumschwimmen. Mikroskopisch erweisen sich die weissen Schollen als vertrocknete, ineinandergedrückte Epidermiszellen; die kleinen Kügelchen bestehen zum grössten Theil aus feinkörnigem Fett und Pigmentdetritus, in dem noch einzelne Umrisse fettig degenerirter oder mit gelben Pigmentkörnchen angefüllter Zellen zu erkennen sind. Die verschiedene Farbe erklärt sich durch reichlicheren Gehalt der braungefärbten Kugeln an gelbem Pigment.

Die Entleerung der Cyste war von günstigem Erfolg auf das Befinden der Patientin; die Schmerzen im Unterleib liessen nach, sie fühlte sich wesentlich erleichtert, Urin und Koth konnte sie spontan lassen. Allein diese Besserung war nur von sehr kurzer Dauer; bereits am anderen Tage musste Patientin wieder katheterisirt werden, da trotz aller Anstrengung kein Tropfen Urin aus der Harnröhre floss, die Temperatur stieg rasch auf 40,3 , bis zum Abend traten 
wieder die heftigsten Sehmerzen im Mastdarme und Unterleibe ein, zugleich bedeutende Auftreibung des Abdomen, rascher, kleiner Puls, belegte Zunge; die Katheterisation wurde immer schmerzhafter und schwieriger.

Eine am 2. September vorgenommene Untersuchung des Unterleibes ergab Folgendes: Ueber der Symphyse fühlt man, giemlich median gelegen, eine cirea kindskopfgrosse Geschwulst von platter Oberfläche, welche nach aufwärts, etwa $2 \mathrm{Cm}$. unterhalb des Nabels, sich nach hinten absenkt, so dass ihre höchste Kuppe wohl beiläufig die Höhe des Nabels erreichen dürfte; zu "den beiden Seiten fithlt man die Grenze der Gesehwulst ungefähr in der Mitte der Poupart'schen Bänder. Die Percussion ergiebt über dem Unterleib tympanitischen Schall, und zwar rechts von der Geschwulst hellen, hohen Düundarmton, oberhalb derselben hellen und vollen, jedoch etwas tieferen Magenton, ïber der Geschwulst gedümpft tympanitischen Ton; die untere Lebergrenze reicht bis zum Rippenbogen, die Milz nicht erheblich vergrössert. Bei Betrachtung des Perineum fällt zunächst auf, dass die von vaxicösen Venen umgrenzte Analöffnung spaltförmig verzogen ist, und von der Mittellinie nach unten und rechts etwa in der Richtung zwischen tuber ossis ischii und Spitze des Steissbeines verläuft; die Perinealfurche ist weniger ausgeprägt, als gewöhnlich. Die Gegend des Cavum ischio-rectale linkerseits ist prall elastisch gespannt, zeigt in der Tiefe deutliche Fluctuation, und zwar lässt sich dieselbe von jener Stelle aus bis zur Gesebwulst oberhalb dẹ Symphyse fortsetzen; die Länge der Geschwulst lässt sich auf $26 \mathrm{Cm}$. schätzen. Bei der Untersuchung per vaginam kommt man mit Mühe mit einem Finger zwischen Symphyse und hintere Scheideawand; aus der Harnröhre ergiesst sich ein Tropfen Blut, aus der Vagina fluor. Dadwrch, dass die ganze Analöffnung weiter herabgedrückt ist, steht das soast im Durchschnitt dreieckige Septum rectow vaginale membranōs verlängert vertikal vor der Analöffnung. Die Entfernung rom hinteren Ende dex Analöffnung zur Steissbeinspitxe beträgt $5,5 \mathrm{Cm}$. Es war somit eine viel bedeutendere Vermehrung: des Umfangs und Spannung der Cyste eingetreten, als vor der Punction, und zwar konnte das rasche Auftreten derselben, der Kleine beschlernigte Puls, das bedentende Fieber, die Schmerzhaftigkeit, keinen Zweifel dariber lassen, daiss eine rapide Vexjauchung, wahrscheinlich auch mit Entwickelung von Gasen, wie der gedämpft tympanitische Schall über der Geschwulst bewies, entstanden sei. Dieser Umstand allein schon, dann aber auch die pralle Spannung der Geschwulst, die jeden Augenblick eine Perforation drohte, die Compression des Mastdarmes, der Vagina und der Harpröhre, stellten die unabweisliche Anzeige zur schleunigen Eröffnung der Cyste and Entleerung des verjauchten Inhalts, - Die Patientin wurde in tiefer Chloroformnarkose in erböhte Steissrückenlage gebracht und die Eröffnung der Cyste durch Professor $\mathrm{C} z \mathrm{rng}$ in folgender Weise vorgenommen: Ein sagittaler cirea $4 \mathrm{Cm}$. langer Schnitt trennte die Weichtheile zwischen Anus und Os sacrum and endigte vor dem letztgenannten 
Punkte in einem Abstande von etwa $3 / 4 \mathrm{Cm}$. Nach Unterbindung mehrerer kleiner Arterien und doppeltseitiger Durchlegung von Fadenschlingen in den freigelegten, weiss glänzenden, derben Cystenbalg, wurde derselbe durch Stich mit einem spitzen. Scalpell eröffnet. Sofort drangen unter flatusähnlichen Geräuschen äusserst stinkende Gase und die schon durch die Punction. bekannten Cystenbestandtheile hervor; die Epidermisschollen hatten theilweise einen solchen Umfang, dass sie die Stichöffnung verlegten und erst nach Entfernung mit dem Finger der Flüssigkeit freien Lauf liessen. Mit einem geknöpften Bistouri wurde dann die Cystenöffnung fast bis zur Länge des Hautschnittes erweitert und nach sorgfälltiger Vereinigung der Cystenund Hautränder der Cysteninhalt soviel als möglich ausgeränmt. Um über völlige Entleerung der Geschwulst sicher zu sein, wurde dann in das Rectum mit der ganzen Hand eingegangen: von der Geschwulstmasse war nirgends mehr etwas zu fühlen, die Compression von Rectum und Vagina und ihr Angedrängtsein gegen die Symphyse waren mit dem Zusammenfaller der Gesehwulst aufgehoben. Leider riss bei der Rectalpalpation die hintere Mastdarmwand bis auf die vordere Cystenwand ein, in einer Länge von circa $4 \mathrm{Cm}$. Der Riss wurde sofort sorgfälltig vernäht, in den leeren Cystenbalg wurden zwei Drainageröhren eingeführt und alle 2 Stunden mit einer Lösung von Kali hypermanganicum durchgespiilt.

Die Spaltung der Cyste war von einer sofortigen Besserung gefolgt; die Schmerzen im Unterleibe liessen nach, die Temperatur erreichte nicht mehr die hohen Zahlen, wie vorher, Harn und Koth konnten spontan entleert werden, das Allgemeinbefinden war zufriedenstellend.

Am 6. September wurde Patientin bei Gelegenheit der Entfernung der Näthe wieder in Steissrückenlage untersucht. Die Umgebung des Afters und der Cystenöffnung ist in mässiger Ausdehnung geröthet und bei Berührung empfindlich. Der Mastdarmriss ist nicht per primam intentionem geheilt; der Cystenbalg ist zum 'Theil mit der äusseren Weichtheilwunde vereinigt, retrahirt sich indess nach Wegnahme der Nähte nicht unbedeutend von dem Hautrande; von der Innenwandung der Cyste lassen sich graue nekrotische Gewebsfetzen abziehen. Am wichtigsten jedoch ist der Befund in der Scheide; beim Einsetzen des Speculum ergiesst sich ein graugrünes, stinkendes, ziemlich reichliches Secret aus ihr; der ganze mittlere Theil der Hinterwand ist mit einem kreisförmigen, fest anhaftenden, gelblichen Belag versehen; ein ebensolcher Belag, wenn auch bedeutend kleiner, findet sich an dem der Symphyse gegenüber liegenden Theile der Harnröhren-Scheidewand. Bei einer drei Tage darauf wieder vorgenommenen Untersuchung fanden sich die nekrotischen Schorfe abgestossen, an der Harnröhre reichte der Snbstanzverlust durch die ganze Dicke der unteren Harnröhrenwand, so dass also eine Harnröhren-Scheidenfistel entstanden war, die eine Lungenausdehnung von etwa $0,5 \mathrm{Cm}$. hatte. Im mittleren Theile der Vagina hatte der Brandschorf gleichfalls an einer Stelle zur tiefgreifenden Ulceration und Perforation ge- 
führt; es führte nämlich eine Fistel, aus der fortwährend Eiter floss, links neben dem unversehrten Rectum vorbei in den Cystenbalg; die Oeffnung war so gross, dass man mit der Spitze des in die Incisionswunde eingefiihrten Zeigefingers bequem in die Vagina gelangen konnte. Es hatte also die Compression der Vagina gegen die Symphyse, durch die starke Ausdehnung der Dermoidcyste, Veranlassung zu gangränösem Decubitus gegeben, welchem an der Stelle des grössten Druckes tiefergreifende Ulceration und Bildung zweier Fisteln, nëmlich einer Harnröhren-Scheiden- und einer (Dermoid-) Cysten-Scheiden-Fistel gefolgt war.

Ueber den weiteren Verlauf des Falles kann ich mich kiurzer fassen. Die Aufgabe der Behandlung bestand darin, für den Abfluss der Wundsecrete und die Reinhaltung der Vagina und des Restes der Cyste Sorge zu tragen; diesen Indicationen konnte man sehr leicht dadurch genügen, dass man ein Drainagerohr von der Incisionswunde dureh die Cysten-Seheiden-Fistel einschob und mehrmals täglich mit einer Lösung von Kali hypermang. durchspülte; an eine Operation der Harnoöhen-Scheidenfistel konnte man jetzt nicht denken, da die Vagina fortwäbrend mit dünnem, jauchigem Eiter angefiillt war und bierdurch eine prima intentio der Anfrischungsränder doch sehr in Frage gestellt worden wäre. Den Cystenbalg selbst musste man der Vereiterung überlassen, da er, vermöge seiner Lage, der weiteren operativen Behandlung nicht zugänglich war. Die Incisionswunde schloss sich bis gegen Mitte November; man konnte sie unbedenklich zuheilen lassen; da der Eiter durch die Vaginalfistel hinreichenden Abfluss hatte und demgemäss keine Retention desselben zu befurchten war. Die Patientin erholte sich sehr rasch und hatte verhältnissmässig wenig subjective Beschwerden, selten, dass sie hier und da über etwas Empfindlichkeit in der linken Unterbauchgegend klagte. Den. Urin konnte sie in der Rückenlage Stunden lang halten, beim Stehen flossen inmer einige Tropfen ab; die Menstruation stellte sich zum ersten Male in Januar wieder ein. Mohrere Injectionen von reiner Jodtinktur, die im Verlaufe des Februar und Marz d.J. gemacht wurden, hatten keine Verminderung der Eiterung zur Folge; erst vom Mai an war dieselbe etwas weniger copiös. Gegen Ende Juni wurde, bei einer aus anderweitigen Griùden vorgenommenen Untersuchung, eine Communication des Cysteurestes mit dem Mastdarme constatirt; die von Granulationen umgebene, etwa stecknadelkopfgrosse Fistelöffnung liegt ă Cm. oberhalb der Analöffnung an der linken Mastdarmwand, es scheint jetzt durch diese neugebildete Perforationsstello die Hauptmasse des Eiters abzufliessen, während durch die Cysten-Scheidenfistel nur wenig mehr abfliesst. Die Verbältnisse für eine Heilung der Harnröhren-Scheidenfistel waren dadureh viel besser gewrorden, so dass man jetzt mit guten Aussichten an die Operation derselben herangehen konnte. Sie wurde am 30. Juni durch Professor Czerny ausgeführt; die Fistelränder wurden flach trichterförmig angefrischt und durch fünf tiefgreifende vähte in der Quere vereinigt. Die Nachbehandlang bestand in täglieh mehrmaliger Catheterisation und einmaliger Ausspuilung der Vagina mit 
warmem Wasser; am 6. Juli wurden die Nähte entfernt und zeigte es sich, dass überall vollständige Verheilung der Ränder eingetreten war.

Wir haben in Vorstehendem einen Krankheitsfall geschildert, der in zweierlei Rücksicht Beachtung verdient, nämlich in klinischer und anatomischer. - Ein bisher vollständig gesundes Mädchen, dessen Menstruation regelmässig ist und dessen Befinden nichts zu wünschen übrig lässt, wird plötzlich olne irgend welche äussere Ursachen von heftigen Schmerzen im Leibe und mehrmaligem Erbrechen befallen, Koth und Urin können gar nicht oder nur mit grosser Anstrengung entleert werden. Als Ursache dieser auffallenden und von Anfang an so heftigen Krankheitserscheinungen findet man nicht eine der gewöhnlichen Ursachen dieser Symptome, etwa eine Perforation, Peritonitis oder eine incarcerirte Hernie, sondern einen Tumor, der seinen Sitz zwischen Rectum und Kreuzbein hat, bisher latent gewesen ist, und nun plötzlich durch eine rasche und bedeutende Ausdehnung die geschilderten schweren Exscheinungen hervorruft, Nachdem andere Möglichkeiten ausgeschlossen sind, muss man als wahrscheinlichen Charakter der Geschwulst eine Neubildung der Art annehmen, wie, sie zuerst von Lebert mit dem Namen ,Dermoideysten" bezeichnet worden sind, und deren Besonderheit bekanntlich darin besteht, dass sie vollständig abgeschlossene Cysten vorstellen, die auf ihrer Innenfläche eine der äusseren Haut gleiche Organisation zeigen, mit Epidermis, Schweiss- und Talgdrüsen, Haaren und Unterhautfettgewebe; den Inhalt der Cyste bildet ein dicker Brei, der aus abgestossenen Epidermisschollen und Hauttalg besteht; seltener werden auch Haare und Zähne gefunden. - Die Probepunction bestätigt dann auch die Richtigkeit der Annahme einer solchen Cyste. Während aber bisher der Grund der Vergrösserung der Cyste nur in einem einfachen Hydrops zu suchen ist, sehen wir nach der Punction eine wesentliche Veränderung mit dem Inhalt vor sich gehen; es tritt eine rasche und intensive Gasentwickelung und Verjauchung ein, die zu einer bedeutenden Vergrösserung der Cyste, Verdrängung der Nachbarorgane und endlich zu gangränösem Decubitus der Vagina und Harnröhre führt, der seinen Abschluss in Bildung einer Cysten-Sccheiden- und einer Harnröhren-Scheidenfistel findet. Wenn nun auch der Durchbruch der vereiterten Dermoideysten - und zwar beziehen sich die meisten Beobachtungen auf Ovarial-Dermoidcysten - in Nachbarorgane, und in specie auch in die Scheide, zu den häufiger bei diesen Tumoren gemachten Beobachtungen gehört, so ist doch die Bildung einer Harnröhren-Scheidenfistel durch Druck einer hinter dem Rectum gelegenen, colossal ausgedehnten Dernoidcyste eine bisher anderwärts noch nicht beobachtete Entstehungsursache der genannten Fisteln. Die Art, wie dieselbe entstanden ist, ist vollständig analog der gewöhnlichen Ursache: dem Drucke der Geburtszange oder des eingekeilten Kindskopfs entspricht der Druck, welchen die ausgedehnte Cyste durch Hervordrängung der Vagina und Anpressen der Haruröhre gegen die Symphyse hervorgerufen hat. 
Fragt man nun nach dem Grunde der Verjauchung, so liegt allerdings die Vermuthung nahe, dass nach der Punction vom Rectum aus durch den Stichkanal Koththeilchen in die Cyste übergetreten seien, welche die Ursache der septischen Zersetzung geworden wären; allein diese Veränderung im Cysteninhalt trat schon nach der Punction mit dem feinsten Probetroicart auf, und es ist doch schwer denkbar, dass eine so feine Oeffinung, noch dazu in der Schleimhaut des Darms, sich nicht sofort geschlossen haben sollte. Eine Betrachtung der bei Lebertl) mitgetheilten Fälle lehrt aber bereits, dass eine Dermoidcyste in den seltensten Füllen unverändert bleibt, dass vielmehr in der Regel gerade die im Abdomen befindlichen entweder zur Zeit des Wintrittes der Geschlechtsreife oder während der Gravidität spontan in Vereiterung und Verjauchung ïbergehen, so dass man sich vom klinischen Standpunkte aus wohl für berechtigt halten kann, wegen dieser Eigenschaft dieselben den ,bösartigen Tumoren" beizuzählen. Als verhältnissmässig günstigster Ausgang einer Verjauchung der Dermoidcysten ist dann noch der Durchbruch nach aussen durch die Bauchdecken mit vorheriger Verwachoung mit denselben, oder in ein Nachbarorgan, wie Vagina, Blase, Rectum, Colon, und dann auf indirectem Wege erfolgende Entleerung des Inhalts nach aussen zu betrachten. Immerhin ist aber der Effekt einer solchen Naturheilung ein sehr mässiger. Der Balg bleibt zurïck und vermöge seiner höheren Organisation und seines ziemlich bedeutenden Gefussseichthums nnterhält ex' endlose jauchige Eiterung, die durch die lange Dauer einmal schon die Kräfte des Patienten auf das Aeusserste erschöpft, andererseits aber anch durch den foetiden Geruch, den sie verbreitet und durch die fortwährende Durchnässung das Leben unerträglich macht, im weiteren Verlaufe aber, selbst wenn der Eiter nach aussen fireien $\mathrm{Ab}$ fluss hat, noch immer neue Perforationen erzeugen kann, wie in unserem Falle nach zehnmonatlichem Bestehen noch in das Rectum. Die Gefahr eines weiteren Durchbruchs, etwa in die Peritonäalhöhle, ist hierbei auch jetzt noch vorhanden. In Hinsicht auf die Therapie der fraglichen Tumoren dürfte sich also auf Grund der bisher gemachten Beobachtungen empfehlen, nach Feststellung der Diagnose iiberall, wo es der anatomische Sitz der Gesehwulst erlaubt, die vollständige Exstirpation derselben yorzunehmen, selbst wenn im gegebenen Augenblicke noch keine drohenden Symptome vorhanden sein sollten, da doch früher oder spater eine spontane Vereiterung mit Sicherheit zu erwarten sein dürfte. Selbst wenn dieser letztere Umstand bereits eingetreten sein sollte, so ist hieraus noch keine Gegenindication für die Radical-Operation einer im Abdomen befindlichen derartigen Geschwulst herzuleiten, wie ein von Prof. Hegar in der hiesigen gynäkologischen Klinik mit günstigem Ausgang operirter Fall einer verjanchten Ovarial - Dermoideyste beweist, Falls die anatomische Lage die Exstirpation unmöglich macht - wie in unserem Falle - so ist wenigstens für möglichst vollstündige Entleerung des Inhalis durch

1) Prager Vierteljahrsschrift. Bd. XV. Jahrg, 1858. S. 25 ff. 
Schnitt oder Punction zu sorgen, letzteres Mittel verspricht allerdings den geringsten Erfolg, da durch die Canüle die festeren Bestandtheile nicht durchpassiren können. In einem solchen Falle ist auf vollständige Ausheilung immer erst nach sehr langer Zeit zu rechnen. Jodinjectionen, welche von Lebert ${ }^{1}$ ) empfohlen werden, haben in unserem Falle gar keinen Erfolg gehabt.

Die Casuistik der Dermoid-Cysten ist eine ziemlich umfangreiche. Am häufigsten scheinen sie vorzukommen im Ovarium; sie sind aber schon an allen möglichen Stellen des Körpers beobachtet worden; so im Gehirn, in der Nase, Stirn, Blasenwand, am Zungenbein, Oberkiefer, Mediastinum, zwischen Uterus und Rectum, Blase und Rectum. Ein dem unserigen analoger Fall, wo also der Tumor zwischen Rectum und Krenzbein seinen Sitz hat, findet sich in der Literatur nur noch ein einziges Mal verzeichnet. Derselbe findet sich in Guy's Hospital Reports ${ }^{2}$ ) beschrieben von Birkett. Im Jahre $1857 \mathrm{kam}$ eine 25 Jahre alte Frau mit einer Geschwulst am Steissbeine in Guy's Hospital und gab an, dass sie bereits seit zwei Jahren die Hervorragung beobachtet habe. Es zeigte sich in der A.fterkerbe etwas auf die rechte Seite hin eine kleine Geschwulst, und man entdeckte mittels des in den After eingebrachten Fingers in dem lockeren Gewebe zwischen Rectum and innerer Steissbeinfläche einen Körper von ziemlich beträchtlicher Grösse. In der letzten Zeit hatte auch die Kranke daselbst Schmerzen verspürt. Durch einen Einschnitt wurde eine eystoide Geschwulst blosgelegt, die ziemlich weit zwischen Steissbein und Rectum hinaufragte und nach der Exstirpation sich als fibröse, mit atheromatösem Brei erfitillte Cyste auswies. Sie war ïber wallnussgross, die Wunde heilte gut.

Ueber die Zeit der Entstehung solcher Geschwïlste liefert unser Fall keinen Anhaltspunkt; man ist wohl genöthigt, sie für angeboren zu halten, da ihre erste Entwickelung noch nicht beobachtet worden ist.

$\mathrm{Ob}$ vielleicht die Entstehung der Cyste in anserem und in dem Falle von Birkett mit der Luschka'schen Steissdrüse im Zusammenhange steht, lässt sich auch nicht weiter verfolgen.

1) A. a. 0 .

2) 3. Reihe. 1859. Vol. 5. S. 252; citirt bei Braune, die Doppelbildungen und angeborenen Geschwülste der Kreuzbeingegend. Leipzig, 1862. 